\title{
Centralized Mobile Users Assignment Strategies for Energy Management in Het-LTE Networks
}

\author{
Dominique Barth, Amira Choutri, \\ Leïla Kloul \\ Université de Versailles \\ 45, Av. des Etats-Unis \\ 78000 Versailles, France \\ \{barth, cham, kle\}@prism.uvsq.fr
}

\author{
Olivier Marcé \\ Alcatel-Lucent \\ France \\ Olivier.Marce@alcatel-lucent.com
}

\begin{abstract}
Power consumption control paradigm constitutes an incentive solution for the serious energy issues faced by operators. In this work, we investigate two mobile users assignment strategies for energy management in heterogeneous LTE networks. Both strategies are based on greedy algorithms which are of low complexity and allow on-line approaches suitable for femtocells management issue. Moreover, as the femtocells can be switched off and the macrocells have to remain always on, both strategies aim at selecting the femtocells to deactivate. That is, the mobile user may be moved from a femtocell to a macrocell or the other way around. The move is legitimate if the power consumption is lower and only if the QoS of the user is not sacrificed. Using simulation and real network data, we assess the performances of proposed strategies, in terms of energy consumption and efficiency of the network, the percentage of served mobile users, the real allocated capacity and the number of deactivated femtocells.
\end{abstract}

\section{Categories and Subject Descriptors}

C.2 [Computer Systems Organization]: COMPUTERCOMMUNICATION NETWORKS

\section{Keywords}

Heterogeneous LTE network, energy consumption optimization, mobile users assignment strategies

\section{INTRODUCTION}

With the considerable growth of mobile operator subscribers and the diversity of the offered services, mobile networks consume more and more energy. Indeed, on the one hand, the mobile terminals (smart-phones) consume energy to handle their spaces (user and system) and their network interfaces (receiving/transmitting)[4]. On the other hand, the base stations of the consume energy for their treatments, like signals reception/transmission from/to their mobile users. Current mobile networks have a real need for power consumption reduction and this for different commercial and ecological reasons. These include a fear of depletion of material resources, global warming, political issues and security of supply problem, and the cost of energy that the combination of these phenomena may increase. Thus network operators are faced with serious energy issues. They must find a compromise between, on the one hand, the QoS that must be offered to their final users, and on the other hand, the cost that allows providing them with more capacity $[1,6]$.

In this paper ${ }^{1}$, we consider heterogeneous LTE networks based on femto and macro base stations. Unlike most previous works (see the related work in Section 2), we focus on reducing the energy consumption in these networks while considering an on-time location of the mobile users. We propose two green models, called FTM-based strategy and MTF-based strategy. The objective of these models is energy consumption optimization while guaranteeing users access control to the network resources. Both strategies are based on greedy algorithms that are of low complexity and allow on-line approaches suitable for femtocells management issue. They both consist of two phases. During the first phase, the mobile users are initially assigned to femtocells (FTM-based strategy) or macrocells (MTF-based strategy). Then in the second phase, each strategy processes a specific load-balancing between the two types of cells. Because the femtocells can be switched off, unlike the macrocells, the purpose of these strategies is to deactivate as many femtocells as possible without overusing the macrocells energy.

Using simulation and real network data, we assess the performances of these strategies, in terms of energy consumption and efficiency of the network, the percentage of served mobile users, the allocated capacity of resource blocks and the number of deactivated femtocells. Through the obtained results we show that both strategies are more efficient compared to other strategies such as the current network operator strategy. Structure of the paper: Section 2 summarizes the related work. Section 3 is dedicated to the network and the implied actors description. Section 4 describes the mobile assignment algorithms and the two energy consumption control strategies that we investigate. Their performances are analyzed in Section 5. Finally, Section 6 concludes the paper.

${ }^{1}$ This work has been partially supported by ANR NetLearn project. 


\section{RELATED WORKS}

Considering that macro base stations must be always on, the objective of mobile operators is to control power consumption of lower part of the network (that is base stations) while guaranteeing QoS to mobile users. Solutions such as the use of renewable energy or the use of more energyefficient equipments have been considered [2, 15]. Clearly these equipments consume less energy than the usual ones but they are more expensive. In the literature, other solutions are investigated. These include the configuration of base stations by adjusting the size of cells according to the traffic [12], or by switching off the base stations, which requires a cooperation between base stations $[2,9,11]$.

Few papers focus on switching off femto base stations instead of macro base stations in heterogeneous LTE networks $[11,8,14]$. Authors in [11] and [8] focus on a daily traffic where their objective was to configure the femto base stations (by switching off) according to the traffic. In [11] base stations switching off is considered to improve the energy efficiency of cellular access networks by reducing power consumption in periods of low traffic. The authors adapt sleep mode schemes of macro base stations in homogeneous networks. They also study the case of several permitted switch offs per day to reduce progressively the number of active base stations. The model proposed in [11] considers that the energy consumed by macro base stations is constant, which is unrealistic [16] as it depends, linearly, on the traffic load. This one varies according to the daily traffic from the femtocells that are switched off.

In [8], authors take into account different sizes of base stations (macro, micro, pico, ...) which have different power consumption profiles. Macro base station power consumption is considered as varying with the load, and the communication through different sizes of base station has different radio resource utilization. The authors introduce two sleep algorithms (single and multiple sleeps) to determine the time instant to enable small base stations sleep mode. They prove that switching off a small base station at a specific time does not decrease the power consumption. This model does not guarantee an energy consumption optimization because when a base station (macro or small) is fed to satisfy the load of the sleep base station, it may consume more power and the gain will be negative.

In [14], authors model the system as a discrete-time Markov process to control the switch on/off of femto base stations. This model depends only on the current state of the system and the available information such as user equipment localization and current femto base station load.

The key factor that impact power consumption of base stations is the traffic load. Most of previous work concentrate to switch off femto base stations during low load traffic in order to not sacrifice mobile users access and guarantee their QoS. In this paper, we manage the traffic based on mobile users assignment control to reduce the power consumption of the network. Note that, current assignment strategies are based on the signal quality report a terminal sends to select the closest cell to which the user is assigned [18]. Moreover in literature, several cell selection techniques based on the Reference Signal Received Power (RSRP) are also proposed for small cells in LTE networks [7].

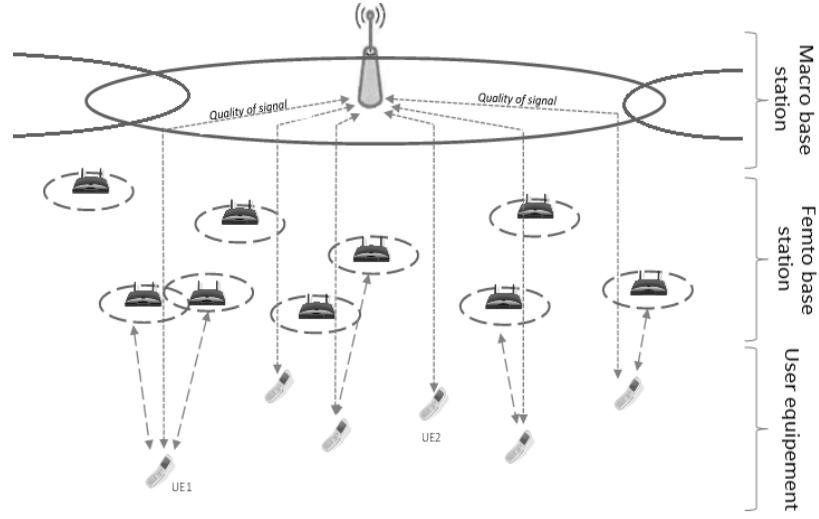

Figure 1: Heterogeneous LTE architecture: a mobile user can detect macrocells (UE2) only, or macrocells and femtocells (UE1).

\section{THE NETWORK DESCRIPTION}

We consider an heterogenous LTE cellular network composed of classical macro base stations and femto base stations. In such networks, outdoor femtocells are deployed by operators to locally increase the capacity of their mobile networks or the operators could rent a partial usage of private femtocells.

Within the coverage area of each macro base station, a set of femtocells is deployed by the same mobile operator. The femto base stations are connected to the macro base stations via the LTE logical X2 interface [1]. In this case, a mobile terminal in the network is able to detect macrocells only, or macrocells and the femtocells in their coverage areas (see Figure 1). Such a network is deployed in urban areas and its objective is to guarantee data communications with a high Quality of Service (QoS) $[1,6]$.

Within the heterogeneous LTE cellular network, three main actors can be identified: the mobile user, the femtocell and the macrocell.

\subsection{The mobile user}

Consider a user $u$ located in the area of a femtocell $f$; we denote by $\operatorname{CovMob}(f)$ the set of all users covered by $f$. Let us denote by $F e m t o(u)=\{f / u \in \operatorname{CovMob}(f)\}$ the set of all femtocells $f$ such that $u$ is in $\operatorname{CovMob}(f)$. We also denote by Connect $(u)$ the cell (femto or macro) to which $u$ is really connected to, that is the cell which really serves the user. This cell can be one of the following cell types:

- a femtocell in Femto $(u)$,

- a macrocell $m$ covering at least one femtocell in Femto $(u)$, that is Connect $(u)=m$,

- nothing, that is $\operatorname{Connect}(u)=\varnothing$.

A user $u$ is characterized by its resource requests $\operatorname{Req}(u)$, in terms of Resource Blocks (RBs) (considered as a capacity value $\operatorname{Capa}(u)$ in the following) [18].

\subsection{The Femtocell and the Macrocell}

A femtocell $f$ can be characterized by its state $S t(f) \in\{O N, O F F\}$, and the sets of users that are potentially or physically connected to it. These sets are, respectively, defined as $U \log (f)=\{u / f \in$ Femto $(u)\}$ and 
Fconnect $(f)=\{u / f=\operatorname{Connect}(u)\}$, and such that, Fconnect $(f) \subseteq U \log (f)$. We also denote by $M a c(f)$ the set of all macrocells covering $f$.

A macrocell $m$ is characterized by the set of all users terminals connected to it, $M$ connect $(m)=\{u / m=\operatorname{Connect}(u)\}$. It covers a set of femtocells noted $\operatorname{CovFem}(m)$.

\subsubsection{Allocation of resources to users}

The whole capacity that a femtocell $f$ (respectively macrocell $m$ ) can provide to connected users is noted Capa $(f)$ (respectively $\operatorname{Capa}(\mathrm{m})$ ). If the capacity requested by a user is satisfied by $f$ (respectively $m$ ), then the capacity dedicated by the cell is $\operatorname{Capa}(u)=\operatorname{Req}(u)$ (respectively

Capa $(u) \geq \operatorname{Req}(u))$. Indeed for femtocells, we consider that the capacity to be allocated is equal to the one requested, but for macrocells, the capacity dedicated to a mobile user $u$ by a macrocell $m$ depends on the capacity requested by $u$, $\operatorname{Req}(u)$, and on the signal quality between the base station of this cell and $u$. This quality is mainly function of the real distance between $u$ and the center of $m$. Worse is this quality, greater is $\operatorname{Capa}(u)$.

To model such a phenomenon, each femtocell $f$ is characterized by a signal quality $\operatorname{Qual}(f, m)$ between $f$ and the base station of each macrocell $m \in \operatorname{Mac}(f)$. This corresponds to the exact position of $f$ in the coverage area of $m$. We consider three situations of a user $u$ in a macrocell $m$, that is, $u$ can be close (good quality $\operatorname{Qual}(u, m)=0)$, far $(\operatorname{Qual}(u, m)=1)$, or very far $(\operatorname{Qual}(u, m)=3)$ from the macrocell base station. Thus the distance MinQual $(u, m)$ between a mobile user $u$ and the base station of the macrocell $m$ covering it is the following:

$$
\operatorname{MinQual}(u, m)=\min _{f \in F e m t o(u) / m \in \operatorname{Mac}(f)} \operatorname{Qual}(f, m)
$$

Therefore the actual capacity provided by the cell is:

$$
\operatorname{Capa}(u)=\operatorname{Req}(u) \times(1+\operatorname{MinQual}(u, m))
$$

\subsubsection{Energy consumption}

With each femtocell $f$, we associate an energy cost function CostFemto $(f)$. This cost depends on the state $(O N$, $O F F$ ) of the cell as follows:

- if the femtocell is deactivated, that is $S t(f)=O F F$, then CostFemto $(f)=0$,

- if the femtocell is activated $(S t(f)=O N)$ but no user is connected to it, then we assume $E_{0}^{f e m t o}$ the power consumption of an empty femtocell,

- if the femtocell is activated and serving users, then

$$
E^{f}=\mathcal{C}_{1}(\mid\{u / \text { Connect }(u)=f\} \mid)+E_{0}^{\text {femto }}
$$

where $\mathcal{C}_{1}$ is a linear function [16].

Regarding each macrocell $m$, we denote by $\operatorname{Load}(m)$ the load of $m$

$$
\operatorname{Load}(m)=\frac{\sum_{u / \operatorname{Connect}(u)=m} \operatorname{Capa}(u)}{\operatorname{Capa}(m)}
$$

This basic load depends on the total capacity of all users that are connected to $m$ and the minimum signal quality they require. More details about the load are provided in the following section.
Assuming that the power consumed by an empty macrocell is $E_{0}^{\text {macro }}$, that is, when no users are physically connected to it, its power cost is the following:

$$
E^{m}=\mathcal{M}_{1}(\operatorname{Load}(m))+E_{0}^{\text {macro }}
$$

where $\mathcal{M}_{1}$ is a linear function and $E_{0}^{\text {macro }}$ is a real number [16].

\section{ENERGY CONSUMPTION CONTROL STRATEGIES}

The control of energy consumption in heterogeneous LTE networks relies on the mobile users assignment control. Given a set of users $U$, each user $u \in U$ can be connected to a cell in Femto $(u)$ or in $M a c(u)=\bigcup_{f \in F e m t o(u)} \operatorname{Mac}(f)$. Thus for each user $u \in U$, we consider two energy consumption control strategies. Both strategies consist of two phases. The first phase executes a greedy algorithm assigning $u$ to a femtocell (femto greedy algorithm) or a macrocell covering it (macro greedy algorithm). The second phase applies a load balancing between the macrocells and the femtocells to minimize the energy consumption.

In the following, we first focus on the greedy algorithms presentation, then describe the two phases of both energy consumption control strategies.

\subsection{The greedy algorithms}

Because the power consumption of a femto base station is less sensitive to the load than the power consumption of a macro base station, two main principles are considered, one for each type of cell:

- Macrocells: try to not fully fill in these cells in order to be able to use all of them during the load balancing phase. Clearly, this takes into consideration the extra cost due to the signal quality.

- Femtocells: fill in as much as possible the femtocells to which, at least, one mobile user is already connected, in order to maximize the number of deactivated femtocells.

Note that a cell is said full if its available capacity does not allow serving a new mobile request.

A mobile user assignment to a femtocell or a macrocell comes down to select the cell (femto or macro) that allows satisfying the cell type corresponding principle (see above). The corresponding optimization problem can also be considered as a set cover problem with capacities and unsplittable demands [5] or as a resource assignment problem [3]. But the specific capacity constraints in our context make the approximation algorithms proposed in the literature for these problems difficult to adapt here. Furthermore, an optimal solution for these problems may not be realistic considering the short sojourn time of a mobile user in a cell and its speed to cross a femtocell. This is why we consider here greedy algorithms with low time complexity.

Indeed, greedy algorithms are of low complexity and allow on-line approaches suitable for femtocells management issue. Such approaches are thus compatible with user mobility between cells. In the following, we present the two greedy algorithms we consider. 


\subsubsection{Femto greedy algorithm}

The first greedy algorithm assigns each mobile user $u \in U$ to a non saturated femtocell $f \in$ Femto $(u)$. If a mobile user cannot be assigned to one of the femtocells, because either it is not possible or the choice is to keep some femtocells off, the algorithm tries to assign it to a macrocell $m \in \operatorname{Mac}(u)$. In our context, the best femtocell is the one that allows minimizing the number of non full femtocells. These cells are the ones having at least one mobile in service.

The purpose of this algorithm is thus not to assign all mobile users in $U$ to femtocells, but to find a good compromise between the number of activated femtocells and the number of mobile users they serve. More precisely, if $U_{n}$ is the set of mobile users that are not assigned yet, the algorithm relies on a partition of the femtocells set $F$ into three subsets possibly updated after each user assignment:

- $F_{0}$ : the subset of empty femtocells which have, at least, one unassigned user in their coverage area. That is, for each femtocell $f \in F_{0}$, there exists $u \in U_{n}$, such that $f \in \operatorname{Femto}(u)$.

- $F_{1}$ : the subset of non full femtocells which have, at least, one unassigned mobile in their coverage area. Initially all the femtocells are empty, then $F_{1}$ is initialized with $20 \%$ of the femtocells in $F_{0}$. This value is chosen experimentally and is preserved when $F_{1}$ is updated with the femtocells having the maximum number of unassigned users in their coverage area.

- $F_{-1}$ : the subset of saturated femtocells and/or having only assigned mobile terminals in their coverage area.

At each step of this algorithm, we consider in priority the unassigned users detecting at least one femtocell in $F_{1}$ (i.e. $F_{1} \cap \operatorname{Femto}(u) \neq \emptyset$ ) and one femtocell in $F_{0}$. Among these users, we select one of those detecting less femtocells in $F_{1} \cup F_{0}$. This mobile user is then assigned to a femtocell in $F_{1} \cap$ Femto $(u)$ that has the maximum number of unassigned mobile users in its coverage area.

When a mobile user is covered by only femtocells belonging to $F_{1}$ (or to $F_{0}$ ), this user is assigned to a femtocell in $F_{1}$ (or in $F_{0}$ ) which has the highest number of unassigned mobile users in its coverage area.

Once the mobile is assigned to a femtocell in $F_{1}$ or $F_{0}$, the capacity of this femtocell is updated. Set $F_{1}$ is also updated, and if necessary, $F_{-1}$ too. In this case, we consider that as long as $\left|F_{1}\right|<\rho \times\left|F_{1} \cup F_{0}\right|$, where $0<\rho \leq 1, F_{1}$ is increased with a femtocell from $F_{0}$ that maximizes the number of unassigned mobile users in its coverage area.

The algorithm ends when mobile user assignment is no more possible.

\subsubsection{Macro greedy algorithm}

In order to maximize, at each step, the number of non full macrocells, we consider a greedy algorithm which consists of assigning each user to a macrocell chosen in a subset of nonsaturated macrocells. Referring to the set cover approach, the algorithm relies on the number of unassigned mobiles in the macrocell coverage area, that is $|\operatorname{Macro}(u)|, u \in U$. The purpose of this greedy algorithm is to find a good compromise between the available capacity of macrocells and the number of users they serve. For that, this algorithm relies on a partitioning of the macrocells set $M$ into two subsets:
- $M_{1}$ : the subset of non saturated macrocells which have, at least, one unassigned mobile user in their coverage area.

- $M_{-1}$ : the subset of saturated macrocells and/or having only assigned mobile users in their coverage area.

At each step of this algorithm, we consider an unassigned mobile user $u \in U_{n}$ detecting at least one macrocell in $M_{1}$, that is, $M_{1} \cap \operatorname{Macro}(u) \neq \emptyset$. This mobile is then assigned to a macrocell $m \in M_{1} \cap \operatorname{Macro}(u)$ that has the minimum number of unassigned mobile users in its coverage area.

Once the mobile user is assigned to a macrocell in $M_{1}$, the capacity of this one is updated. Set $M_{1}$ is also updated, and if necessary, $M_{-1}$ too. When no more mobile user assignment is possible, the algorithm ends.

\subsection{The power consumption control strategies}

We define now the two strategies we consider to control the power consumption in the network. Both strategies consists of two phases. The first phase (Phase 1) executes one of the greedy algorithms defined above. Then, to minimize the whole energy consumption, we consider a load balancing phase (Phase 2) between macrocells and femtocells. This last phase consists mainly in deciding which femtocells to deactivate by reallocating the users connected to them to macrocells, if the energy over cost (depending on the signal quality parameter) is not too high.

\subsubsection{The FTM-based strategy}

The first energy consumption control strategy consists of the following two consecutive phases:

- Phase 1: Execute the femto greedy algorithm defined in Section 4.1.1). At the end of this phase, the remaining unassigned mobile users are considered for assignment to the macrocells in which they have the best signal quality, that is, macrocells in which they are the closest to the base station.

- Phase 2: In order to deactivate a maximum number of femtocells, the second phase of this strategy consists in transferring the mobile users from femtocells to the macrocells overlapping them and having enough capacity to absorb these mobiles. This transfer process is noted FTM (Femto To Macro). Empty femtocells can then be switched off. However, the femtocells that are deactivated, in priority, are the ones providing the maximum energy saving. The selection is done according to the following procedure:

(a) Compute the energy consumption gain of each femtocell $f \in\left(F_{-1} \cup F_{1}\right)$ as follows: if the macrocells in $\operatorname{Mac}(f)$ have globally enough available capacity to absorb all the mobile users in $f$, we compute the energy consumption, $E C(m)$, of each macrocell $m \in \operatorname{Mac}(f)$ when serving the transferred mobiles from $f$. The gain is then the difference between the energy consumption of $f$ and the minimum energy cost of the mobile users transfer from $f$ to macrocells in $M a c(f)$. Note that this gain can be negative.

(b) Select the femtocell $f$ with the best positive gain, if it exists. Its mobile users are then transferred 
to the macrocells in $M a c(f)$ to which they will be effectively connected. $f$ is deactivated and these macrocells capacities are updated.

The FTM process ends when there is no more positive energy gain.

In the rest of the paper, FTM-based strategy is referred to as strategy $S_{1}$.

\subsubsection{The MTF-based strategy}

The second and last strategy consists of the following two phases:

- Phase 1: execute first the macro greedy algorithm defined in Section 4.1.2. Once it is no more possible to assign mobiles to macrocells, the remaining unassigned mobiles are considered for assignment to femtocells based on the signal quality at which the femtocells are detected.

- Phase 2: It consists of transferring the mobile users from macrocells to non full femtocells. The objective of this process, which is noted MTF (Macro To Femto), is to transfer the mobile users of a macrocell $m$ to the femtocells that $m$ overlaps. This can be possible only if the capacity of the femtocells allows absorbing the macrocell traffic. The femtocells that are selected to receive the macrocell customers, in priority, are the ones providing maximum energy consumption decrease. Thus the femtocells selection procedure is the following:

(a) For each mobile user $u$ in macrocell $m$, $m=$ Connect $(u)$, compute the energy consumption gain of each neighboring femtocell $f$, $\operatorname{Mac}(f) \subset \operatorname{Mac}(u)$, as follows: if a femtocell

$f \in$ Femto $(u)$ has enough available capacity to serve $u$, the gain in this case is the difference between the energy consumption of $m$ and $f$ in both phases. Formally, if $C\left(u_{f}\right)$ and $C\left(u_{m}\right)$ are the energy cost if mobile user $u$ is assigned to femtocell $f$ and macrocell $m$, respectively, $f$ is selected if $C\left(u_{f}\right)<C\left(u_{m}\right)$.

(b) Consider the femtocell $f \in$ Femto $(u)$ with the maximum positive gain, if it exists, that is, $C\left(u_{f}\right)=\min _{f_{i} \in \text { Femto }(u)} C\left(u_{f_{i}}\right)$, mobile user $u$ is effectively assigned to femtocell $f$. The cells capacities are updated accordingly.

The MTF transfer process ends when all initially assigned mobiles to macrocells during Phase 1 are revisited and no more energy saving is possible. Empty femtocells can then be deactivated.

In the rest of the paper, MTF-based strategy is referred to as strategy $S_{2}$.

\section{PERFORMANCE ANALYSIS}

In order to assess the efficiency of both energy consumption control strategies, we compare the performances of each strategy $S_{i}, i \in\{1,2\}$ to the performances of an approach $R_{i}$ in which the first phase consists of an initial assignment

\begin{tabular}{|c|c|c|c|}
\hline \multicolumn{2}{|c|}{ Strategy } & $\begin{array}{l}\text { Phase } 1 \\
\text { Initial mobiles assignment }\end{array}$ & $\begin{array}{l}\text { Phase 2 } \\
\text { mobiles trans- } \\
\text { fer (effective } \\
\text { connection) }\end{array}$ \\
\hline \multicolumn{2}{|c|}{$S_{1}$} & $\begin{array}{l}\text { femto greedy algorithm then } \\
\text { closest macrocells for the re- } \\
\text { maining mobiles }\end{array}$ & $\begin{array}{l}\text { as defined in } \\
\text { FTM strategy }\end{array}$ \\
\hline \multicolumn{2}{|c|}{$S_{2}$} & $\begin{array}{l}\text { macro greedy algorithm then } \\
\text { closest famtocells for the re- } \\
\text { maining mobiles }\end{array}$ & $\begin{array}{l}\text { as defined in } \\
\text { MTF strategy }\end{array}$ \\
\hline \multicolumn{2}{|c|}{$R_{1}$} & $\begin{array}{l}\text { closest femtocells then clos- } \\
\text { est macrocells for the re- } \\
\text { maining mobiles }\end{array}$ & $\begin{array}{l}\text { as defined in } \\
\text { FTM strategy }\end{array}$ \\
\hline \multicolumn{2}{|c|}{$R_{2}$} & $\begin{array}{l}\text { closest macrocells then clos- } \\
\text { est femtocells for the re- } \\
\text { maining mobiles }\end{array}$ & $\begin{array}{l}\text { as defined in } \\
\text { MTF strategy }\end{array}$ \\
\hline \multirow[t]{2}{*}{$\mathrm{BS}$} & 1 & $\begin{array}{l}\text { - random cell type (femto or } \\
\text { macro) then the closest cell }\end{array}$ & \multirow[t]{2}{*}{ - } \\
\hline & 2 & $\begin{array}{l}\text { - random cell type (femto or } \\
\text { macro) then the closest cell } \\
+ \text { deactivate empty femto- } \\
\text { cells }\end{array}$ & \\
\hline
\end{tabular}

Table 1: Considered scenarios

of a mobile user to a detected cell (femtocell for $i=1$ and macrocell for $i=2$ ), based on a random choice simulating radio signal measurements.

We also compare the performances of each strategy $S_{i}$, $i \in 1,2$, to a Basic Strategy (BS), in which the type of cell (femtocell or macrocell) a mobile user can be assigned to is randomly (uniformly) chosen. In this case, if the mobile is assigned to a femtocell (respectively a macrocell) then its terminal is connected to the closest femtocell (respectively macrocell). In the following, we note $B S_{1}$ this strategy and $B S_{2}$ the same strategy where empty femtocells are deactivated and this to be in line with the approaches in the literature $(([8][11][14]))$. Note that these approaches do not apply any users assignment control. Table 1 summarizes all considered scenarios.

Remember that in current LTE Het-Nets femto base stations are deployed to help the macrocell-based network to face some of its problems [10]. While in this paper, we consider femtocells and macrocells equally in the network.

All approaches are assessed using simulation which results are obtained for a confidence level of $95 \%$.

\subsection{Building the network coverage}

We consider field data from cellular networks of Houston,Texas, a major US urban area [13]. The data sets were gathered on mobile terminals belonging to members of the Rice community. The data collection lasted between three and six weeks, depending on the participants. We deduce the mobile network architecture which consists of one hundred (100) femtocells and thirty-six (36) macrocells. Using the collected data sets, we extract the neighborhood of each cell in the network. This provides us with the set of femtocells that are deployed within the coverage of each macrocell. Additionally, we assume the existence of an umbrella macrocell, that is, a macrocell that can absorb the traffic in the areas that are not covered with regular macrocells.

In our experiments, we define a femtocell capacity of 16 


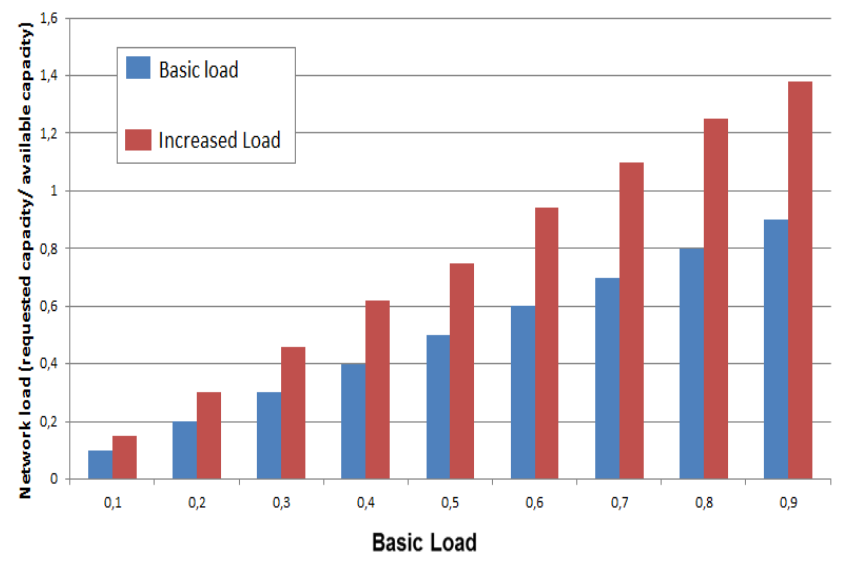

Figure 2: Network load versus basic and increased loads

mobile users. The macrocells capacity is set to $14 k$ of RBs while a mobile user request is set to $10 \mathrm{RBs}$.

The energy consumption of the base stations of both the femtocells and the macrocells are load dependent and are respectively:

- $E^{f}=E_{0}^{\text {femto }}+0.8 W \times \operatorname{Load}(f)$

- $E^{m}=E_{0}^{\text {macro }}+188 W \times \operatorname{Load}(m)$

where $E_{0}^{\text {femto }}=9.6 \mathrm{~W}$ and $E_{0}^{\text {macro }}=260 \mathrm{~W}$ are the power consumption of empty femtocells and macrocells, respectively [16].

The load of the network can basically be defined as the sum of RBs requested by all mobile users divided by the sum of capacities of all macrocells and femtocells. We refer to this load definition as the basic load (see Equation 3). But this definition does not take into account the fact that the number of RBs that could finally be allocated to a user assigned to a macrocell can be greater than the number of RBs it requests, depending on the signal quality (related to the corresponding power boosting level [16]). In our model, the signal quality is directly function of the distance between the mobile user and the base station of the macrocell. Thus for each mobile user covered by, at least, one macrocell, we consider the one in which the signal quality is the best for this user and we compute the required number of RBs to be allocated by the macrocell to satisfy it (for users covered by any femtocell, we keep considering the number of requested RBs). Based on this number of RBs per user, we consider a load defined as the increased load. The difference between the basic load and the increased load provides an idea about the possible impact of the user assignment on the real load, compared to the basic load. Note also that these load definitions do not take into account the distribution of loads over all cells.

Figure 2 shows the evolution of the interval between basic and increased loads for different values of the basic load, based on 200 networks and 50 instances for each one.

\subsection{Numerical results}

To assess the efficiency of our energy consumption control strategies, we investigate the following performance measures:
- the energy consumption of the network,

- the percentage of activated femtocells,

- the percentage of satisfied mobile users, that is, mobile users which receive the minimum requested quantity of RBs,

- the percentage of allocated RBs of the network,

- the relative energy efficiency of the network (Eff). This one is defined as the average network energy consumption per served mobile user.

1. Energy consumption of the network: Figure 3 shows the results we obtain for all strategies as functions of both the basic and increased loads. In particular, it shows the importance of controlling the mobile assignment for the energy consumption of the network; the lack of such a control as in the first basic strategy $\left(B S_{1}\right)$ leads to a high energy consumption and this for all network loads.

A simple switch off of the femtocells $\left(B S_{2}\right)$ allows already reducing this consumption, in particular when the network is not loaded (about $7 \%$ reduction). This is how some related works proceed $([8,11])$. But it is more gainful if a mobile assignment control is considered. An assignment control like in strategy $S_{2}$ allows a further reduction of the energy consumption of the network, and this for all load values.

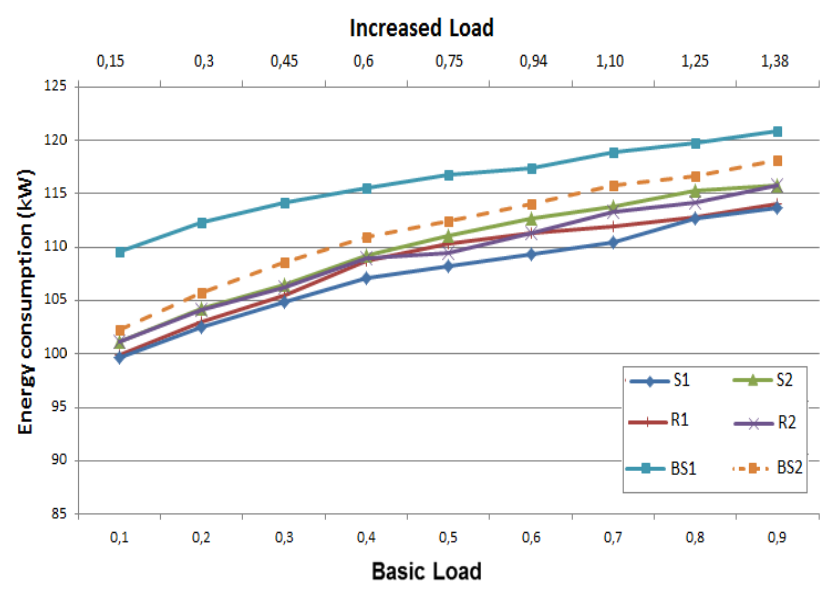

Figure 3: Energy consumption of the network.

Comparing strategies $S_{1}$ and $S_{2}$, we can see that the former provides better results for all loads. However as the load (basic and increased) decreases, the difference decreases also due to the fact that the second phase of both strategies is based on a load balancing criterion. With $S_{1}$, when the network is not loaded (macrocells have more available capacities), more femtocells can be offloaded to the macrocells covering them.

$S_{2}$ and $R_{2}$ assign mobile users to macrocells in priority. Knowing that the large quantity of energy is consumed by macrocells explains why strategies cannot enhance more the energy consumption of the network. Clearly for all loads, strategy $S_{1}$ provides better performances in terms of energy consumption. However, when the network is unloaded ( $b a$ sic load $\leq 0.3)$, all the strategies, but $B S_{1}$, behave pretty similarly. 
To summarize, in terms of energy consumption, giving priority to an initial assignment of the users to femtocells during the first phase, and the load balancing during the second phase can enhance the energy consumption of the network even if the assignment is based on signal quality $\left(R_{1}\right.$ and $\left.R_{2}\right)$.

2. Percentage of activated femtocells: Figure 4 shows that $S_{1}$ and $R_{1}$ are the strategies that allow deactivating the highest percentage of femtocells, and this for all loads.

Both strategies give priority to femtocells during the first phase and offload them to the macrocells covering them, if there is a benefit for energy consumption optimization. Giving priority to macrocells during the first phase, like in strategies $S_{2}$ and $R_{2}$, activates more femtocells. For all network loads, strategies disperse mobile users within the cells.

As there is no control strategy to activate/deactivate femtocells in Het-LTE currently, all femtocells are activated in the basic strategy $\left(B S_{1}\right)$. Regarding basic strategy $B S_{2}$ in which empty femtocells are deactivated, when the network is not very loaded, it provides the same results as strategies $S_{2}$ and $R_{2}$ which have mixed curves. However as the load increases, the pourcentage of deactivated femtocells by $B S_{2}$ decreases, compared to these strategies.

Figure 3 and Figure 4 show also that femtocells deactivation can be the key factor to energy consumption improvement of the network. Strategies $S_{1}$ and $R_{1}$ performances prove it.

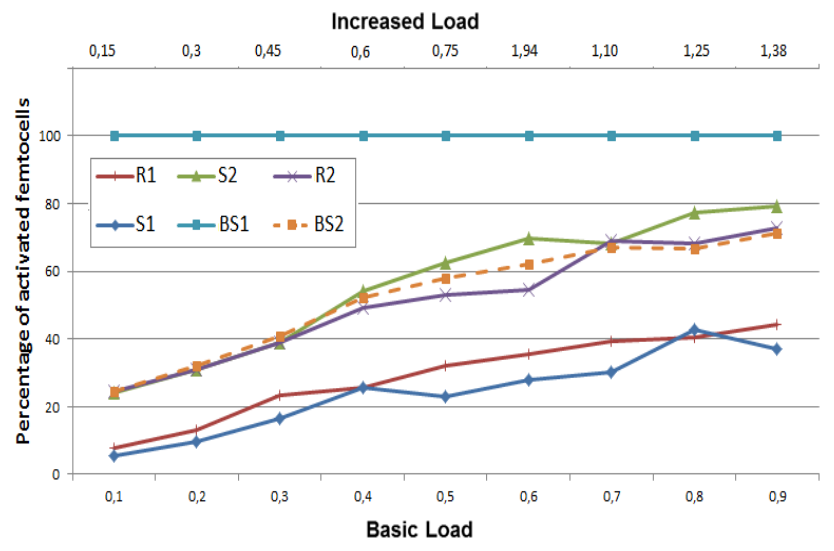

Figure 4: Percentage of activated femtocells.

3. Percentage of satisfied mobile users and allocated RBs: Figure 5 shows once again that strategies $S_{1}$ and $R_{1}$ are more efficient, in terms of the percentage of served users when the network is lightly loaded (increased load less than $75 \%$ ). They are closely followed by strategies $S_{2}$ and $R_{2}$. When the network load increases, $B S$ strategies serve less mobile users (note that $B S_{1}$ and $B S_{2}$ curves are mixed up).

This figure shows also that the percentage of served mobile users decreases as the loads increase for all strategies, and especially when $S_{1}$ or $R_{1}$ is used. Indeed, the FTM-based strategy assigns mobile users to femtocells in priority and this one has a limited capacity than macrocells.

When the network is heavily loaded (95\% of increased load and more) the $B S$ strategies cannot serve more than $55 \%$ of mobile users, and the other strategies serve between $65 \%$ and $60 \%$ of total satisfied users.

Clearly, the fact that the strategies make energy consump-

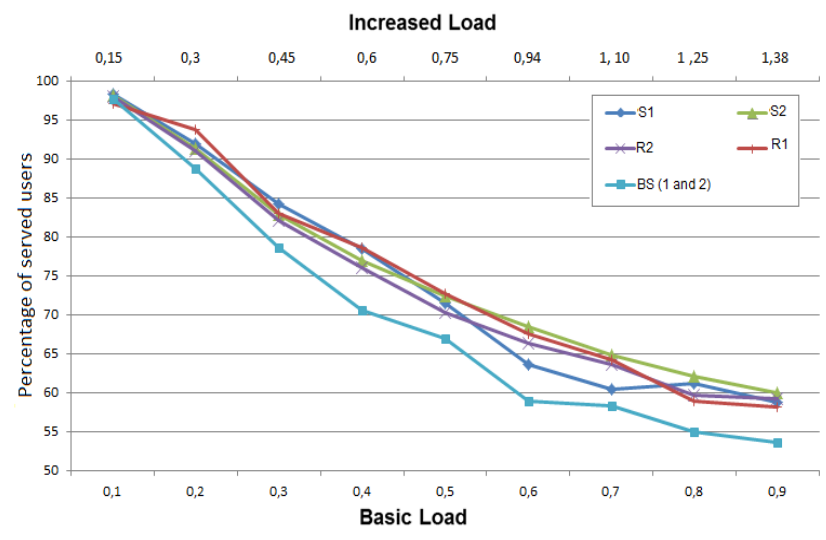

Figure 5: Percentage of satisfied mobile users.

tion savings does not have an impact on the percentage of served mobile users and the offered QoS. Quite the opposite, it enhances the percentage of satisfied mobile users by managing RBs allocation. Indeed, Figure 6 proves that the key factor to serve more mobiles while consuming less energy is the mobile users assignment control.

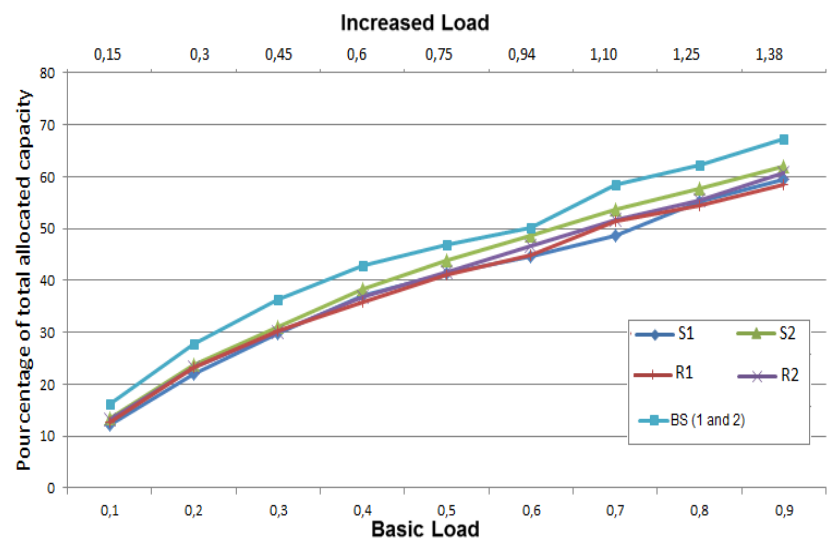

Figure 6: Percentage of total allocated capacity

Because of the signal quality impact on resource allocation, some mobile users monopolize more capacity if they are connected to a macrocell. Indeed, the allocated capacity is greater than the requested one. Considering strategies $S_{2}$ and $R_{2}$, the mobile users are assigned to macrocells in priority, which is more capacity consuming. This reduces the percentage of served mobile users most of the time. For this reason, strategies $S_{1}$ and $R_{1}$, which assign the mobiles to the femtocells in priority, serve more mobile users while allocating less RBs. Figure 5 and Figure 6 show that this property is especially true in this case when the network becomes loaded (increased load at 90\%).

4. Relative energy efficiency: Figure 7 shows the ratio between the results in Figure 3 and Figure 5. Lower is the efficiency value, much better is the energy consumption. For all network loads, proposed strategies $\left(S_{1}, R_{1}, S_{2}\right.$ and $\left.R_{2}\right)$ enhance the efficiency of the network by controlling mobile users assignment. Indeed, these strategies provide better results than the results obtained using $B S$ approaches $\left(B S_{1}\right.$ 
and $\left.B S_{2}\right)$.

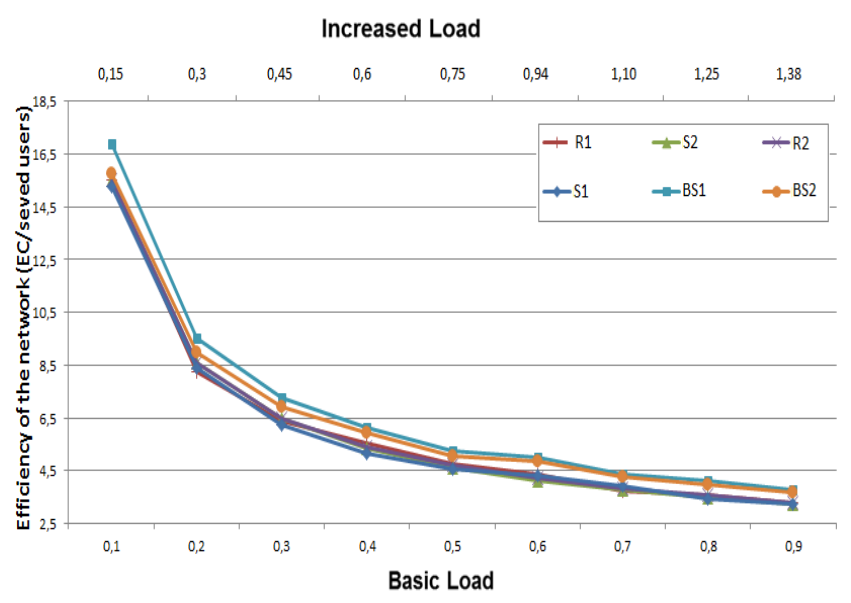

Figure 7: Efficiency of the network $E f f$

5. Global synthesis of the proposed strategies: The main objective of any mobile operator is to maximize the number of satisfied users. Thus, by controlling mobile users assignment, the FTM-based approaches $\left(S_{1}\right.$ and $\left.R_{1}\right)$ are the most efficient in terms of energy consumption and percentage of served mobile users, compared to MTF-based approaches $\left(S_{2}\right.$ and $R_{2}$ ). FTM-based approaches are also more gainful for dimensioning purposes, in terms of required femtocells. These strategies require centralized implementation and offer a global control of the network, which is compatible with today standards of mobile networks. Their low time complexity makes it possible to execute each mobile user location change for reasonably sized network coverage.

\section{CONCLUSION}

In this paper, two strategies are proposed according to mobile users initial assignment priority. Each strategy consists of an initial assignment of the mobile users then offloading them from/to macrocell (resource allocation) if it is gainful, in terms of energy consumption.

Using simulation experiments and real data of network topology, the performances of our strategies are compared to the ones illustrating the strategy used currently by mobile operators and strategy proposed in the literature. Proposed strategies improve energy consumption optimization of the network without any impact on the percentage of mobile users satisfied. Quite the opposite, more mobile users are served when their assignment is controlled. The proposed strategies are centralized and can be considered in a multitenancy architecture to minimize the information exchanges between the base stations [17]. They are compatible with mobility in heterogeneous LTE networks and allow them to evolve as such. In the future, we will focus on real time constraints and more fairness between mobile users.

Acknowledgment: The authors wish to thanks Alberto Conte of Alcatel-Lucent Bells Labs.

\section{REFERENCES}

[1] J. Andrews, H. Claussen, M. Dohler, S. Rangan, and M. Reed. Femtocells: Past, present, and future. IEEE Journal on Selected Area in Communications, 2012.
[2] I. Ashraf, F. Boccardi, and L. Ho. SLEEP mode techniques for small cell deployments. IEEE Communications Magazine, 2011.

[3] Y. Azar, J. Naor, and R. Rom. The competitiveness of on-line assignments. In Journal of Algorithms Volume 18, Issue 2, 1995.

[4] A. Azcorra. Green wireless: Towards minimum per-bit linear energy consumption in wireless communications. In The ACM International Conference on Modeling, Analysis and Simulation of Wireless and Mobile Systems, 2013.

[5] B. Behsaz and M. S. Z. Svitkina. New approximation algorithms for the unsplittable capacitated facility location problem. In Algorithm Theory SWAT, 2012.

[6] M. Z. Chowdhury, Y. M. Jang, and Z. J. Haas. Network evolution and QoS provisionning for integrated femtocell/macrocell networks. International Journal of Wireless \& Mobile Networks, 2010.

[7] T. S. Dhaval M. Tandel. Cell selection techniques in heterogeneous lte advanced system. International Journal on Recent and Innovation Trends in Computing and Communication, 2014.

[8] P. Dini, M. Miozzo, N. Bui, and N. Baldo. A model to analyze the energy savings of base station sleep mode in lte hetnets. In Green Computing and Communications, iThings/CPSCom, 2013.

[9] L. Haratcherev, M. Fiorito, and C. Balageas. Low-power sleep mode and out-of-band wake-up for indoor access points. In IEEE GLOBECOM, 2009.

[10] D. Knisely, T. Yoshizawa, and F. Favichia. Standardization of femtocells in 3gpp. In Communications Magazine, IEEE, 2009.

[11] M. Marsan, L. Chiaraviglio, D. Ciullo, and M. Meo. Multiple daily base station switch-offs in cellular networks. In Fourth International Conference on Communications and Electronics, 2012.

[12] Z. Niu, Y. Wu, J. Gong, and Z. Yang. Cell zooming for cost-efficient green cellular networks. Communications Magazine, IEEE, 2010.

[13] A. Rahmati and L. Zhong. Crawdad data set rice/context (v. 2007-05-23). 2007.

[14] L. Saker, S.-E. Elayoubi, R. Combes, and T. Chahed. Optimal control of wake up mechanisms of femtocells in heterogeneous networks. Selected Areas in Communications, IEEE Journal on, 2012.

[15] A. Spagnuolo, A. Petraglia, C. Vetromile, R. Formosi, and C. Lubritto. Monitoring and optimization of energy consumption of base stations. Energy, 2015.

[16] G. T.-R. WG1. Base station power model. In Release 11, 2011.

[17] Y. Zaki, L. Zhao, C. Goerg, and A. Timm-Giel. Lte mobile network virtualization. In Mobile Networks and Applications, Springer US, 2011.

[18] J. Zyren and W. McCoy. Overview of the 3gpp long term evolution physical layer. In 3GPPEVOLUTIONWP.Rev 0, 2007. 